\title{
Epidemiology, microbiology, and treatment patterns of pediatric patients hospitalized with pneumonia at two hospitals in China: a patient chart review study
}

Therapeutics and Clinical Risk Management

\section{Zehuai Wen' \\ Jia $\mathrm{Wei}^{2}$ \\ Huiling Xue ${ }^{2}$ \\ Yunqin Chen ${ }^{2}$ \\ David Melnick ${ }^{3}$ \\ Jesus Gonzalez ${ }^{4}$ \\ Judith Hackett ${ }^{5}$ \\ Xiaoyan $\mathrm{Li}^{\prime}$ \\ Zhaolong $\mathrm{Cao}^{6}$}

'Key Unit of Methodology in Clinical Research, Guangdong

Provincial Hospital of Chinese Medicine, Guangzhou, ${ }^{2}$ AstraZeneca

R\&D Information China, Shanghai,

China: ${ }^{3}$ Anti-Infectives Actavis, Inc. Harborside Financial Center, Jersey City, NJ, USA; ${ }^{4}$ AstraZeneca, Manchester, UK; ${ }^{5}$ AstraZeneca, Gaithersburg, MD, USA; 'Peking University People's Hospital, Beijing, China
Correspondence: Zehuai Wen Key Unit of Methodology in Clinical Research, Guangdong Provincial Hospital of Chinese Medicine, II I Dade Road, Guangzhou 510120, China Tel +862081887233 Email wenzh@gzucm.edu.cn
Background: The etiology, epidemiology, treatment patterns, and clinical outcomes of neonatal and pediatric pneumonia patients in China are not well reported. This retrospective chart review study aimed to describe such information among neonatal ( 0 to 27 days) and pediatric (28 days to $<18$ years) pneumonia patients in two regions of China.

Methods: Electronic medical records of pneumonia hospitalizations (aged $<18$ years) admitted between 2008 and 2013 from four hospitals under Guangdong Provincial Hospital of Chinese Medicine (Southern China) and between 2010 and 2014 at Peking University People's Hospital (Beijing, Northern China) were reviewed.

Results: The average age of neonatal hospitalizations in Beijing ( $\mathrm{n}=92)$ was 3.5 days. The mean length of hospital stay was 11.2 days, and no deaths occurred. Staphylococcus epidermidis was the most common bacteria found in Beijing patients, whereas Mycoplasma pneumoniae was the most common bacteria found in Guangdong patients. The average age of pediatric hospitalizations was $3.3( \pm 3.1)$ and $6.5( \pm 5.6)$ years in Guangdong $(n=3,046)$ and Beijing $(n=222)$, respectively. The mean length of hospital stay was 17.4 and 5.8 days, and overall mortality rates were $0.2 \%$ and $0.5 \%$.

Conclusion: The findings revealed a low level of bacterial isolation and hence microbiological diagnoses. There was a low level of in-hospital mortality due to pneumonia, and the majority of hospitalizations were discharged from hospital, suggesting that current practice was generally effective. Neonatal hospitalizations were greater than pediatric hospitalizations in Beijing along with disparity in bacterial profile when compared with Guangdong, intending a need to improve neonatal pneumonia prophylaxis and selection of appropriate treatment.

Keywords: pediatric, neonatal, pneumonia, epidemiology, microbiology, antibiotic therapy

\section{Introduction}

Children are the most common victims of pneumonia. Annually, close to 156 million children present with pneumonia within 5 years of birth worldwide. It is estimated that two million cases result in death, of which $\sim 95 \%$ occur in developing countries. ${ }^{1}$ China alone contributes to 21 million pediatric pneumonia cases annually with towering rates of hospitalizations and mortality. ${ }^{2}$ Neonates are at the greatest risk of death from pneumonia ${ }^{3,4}$ as many of the normal lung defenses are not yet fully developed, leading to an increased susceptibility to infection. ${ }^{4}$ Additionally, mortality rates are considerably higher among low birth weight neonates compared with normal birth weight neonates. ${ }^{5}$ The etiology of bacterial pneumonia differs depending on the 
source of infection. Community-acquired pneumonia (CAP) is the single leading cause of death globally in children aged $<5$ years. ${ }^{6}$ The etiological agents implicated in childhood CAP worldwide vary with age. Group B streptococcus and Gram-negative enteric bacteria are the most commonly isolated CAP pathogens in neonates $<3$ weeks old and are often acquired from the mother by vertical transmission. ${ }^{7}$ Streptococcus pneumoniae is the most common etiological agent of CAP in infants aged 3 weeks to 3 months, followed by viral pathogens, ${ }^{8}$ whereas Mycoplasma pneumoniae and Chlamydia pneumoniae are the common bacterial causes of CAP from 4 months to adolescence. ${ }^{7}$

Hospital-acquired pneumonia (HAP) is one of the fatal hospital-acquired infections in the pediatric intensive care unit (ICU) associated with a different epidemiological and etiological profile compared with CAP. Viruses (most commonly respiratory syncytial virus) cause majority of the pediatric HAP cases globally. ${ }^{8}$ Gram-negative bacteria, such as Escherichia coli, Klebsiella pneumoniae, and Pseudomonas aeruginosa, are the predominant causes of HAP. ${ }^{9}$ Early-onset neonatal pneumonia often develops in utero or within the first week of life and is predominantly caused by Gram-negative bacteria. ${ }^{3}$ Gram-negative bacilli are responsible for $30 \%$ of nosocomial pneumonias in neonates. ${ }^{10}$ In contrast, Gram-positive bacteria are responsible for the largest proportion of HAP later in the neonatal period ${ }^{3}$ and many, including methicillin-resistant $S$. aureus (MRSA), coagulase-negative staphylococci (predominantly Staphylococcus epidermidis), and vancomycin-resistant enterococci, are multidrug resistant.

Because of the large number of pathogenic agents that can cause pneumonia in neonates and children, determining the cause in an individual patient may be challenging. Even with the use of sophisticated laboratory techniques, it is not possible to identify a causative pathogen in $25 \%-33 \%$ of pneumonia cases. ${ }^{11}$ Etiological studies of childhood pneumonia are complicated by difficulties in obtaining an accurate microbiological diagnosis, due to the reluctance of physicians to perform invasive procedures such as lung aspiration and bronchoalveolar lavage (BAL) in children, difficulties obtaining adequate sputum samples, and the low yield of pathogens identified from blood cultures. ${ }^{12}$ Such difficulties are exacerbated in the developing world due to limited facilities and resources to perform appropriate specimen collection and testing.

Few recent studies have documented the bacterial etiology of childhood pneumonia in China. S. pneumoniae and Haemophilus influenzae have been implicated in pediatric pneumonia in China; ${ }^{13}$ however, these results were based on the presence of bacteria in the nasopharynx, which may not necessarily be indicative of lower respiratory tract infection, as normal bacterial flora, as well as pneumonia pathogens, are frequently identified. ${ }^{10,11} \mathrm{~A}$ retrospective study on the neonatal mortality rates in China from the last two decades revealed that $4 / 5$ th of the total mortality was due to premature birth, congenital abnormalities, or pneumonia and that the scenario has improved lately in both urban and rural areas since then. ${ }^{14}$ Interhospital variations in prescribing treatments and microbiological diversity may differ among different regions of the country. Hence, a comparative study on the prevalence, microbial profile, prescribing patterns, and hospital diagnosis of different hospitals may be useful for health care professionals and researchers to realize and rectify the gaps in the prevention of the disease and to set novel/updated treatment guidelines. For this purpose, we reviewed patient demographics, microbiological characteristics, treatment patterns, and clinical outcomes of hospitalized neonatal and pediatric patients diagnosed with pneumonia in hospitals of northern and southern China.

\section{Methods}

\section{Study sites, subjects, and design}

This was a retrospective, noninterventional, chart review to assess the epidemiology, clinical management, and outcomes of neonatal and pediatric patients hospitalized with pneumonia, using data from Guangdong Provincial Hospital of Chinese Medicine (CM), Guangzhou, Guangdong Province, China, and Peking University People's Hospital, Beijing, China.

Electronic medical records (EMR) of all unique hospitalizations (admissions to hospital) with an International Classification of Diseases diagnosis code of pneumonia, interstitial pulmonary disease/other lung infection with a positive bacterial culture, or influenza with no virus identified prior to discharge between 2008 and 2013 at the Guangdong hospital and between 2010 and 2014 at the Beijing hospital were reviewed and extracted for analysis.

Study flow chart is depicted in Figure 1. Data for hospitalizations aged $<18$ years are presented. Data from the two study sites were analyzed separately to enable comparison. Neonatal and pediatric data were analyzed separately, due to the inherent differences between these two patient populations. Neonatal hospitalizations were defined as those aged 0 to 27 days, and pediatric hospitalizations were defined as those aged 28 days to $<18$ years. Data for adult hospitalizations aged 18 years or older will be reported separately. 


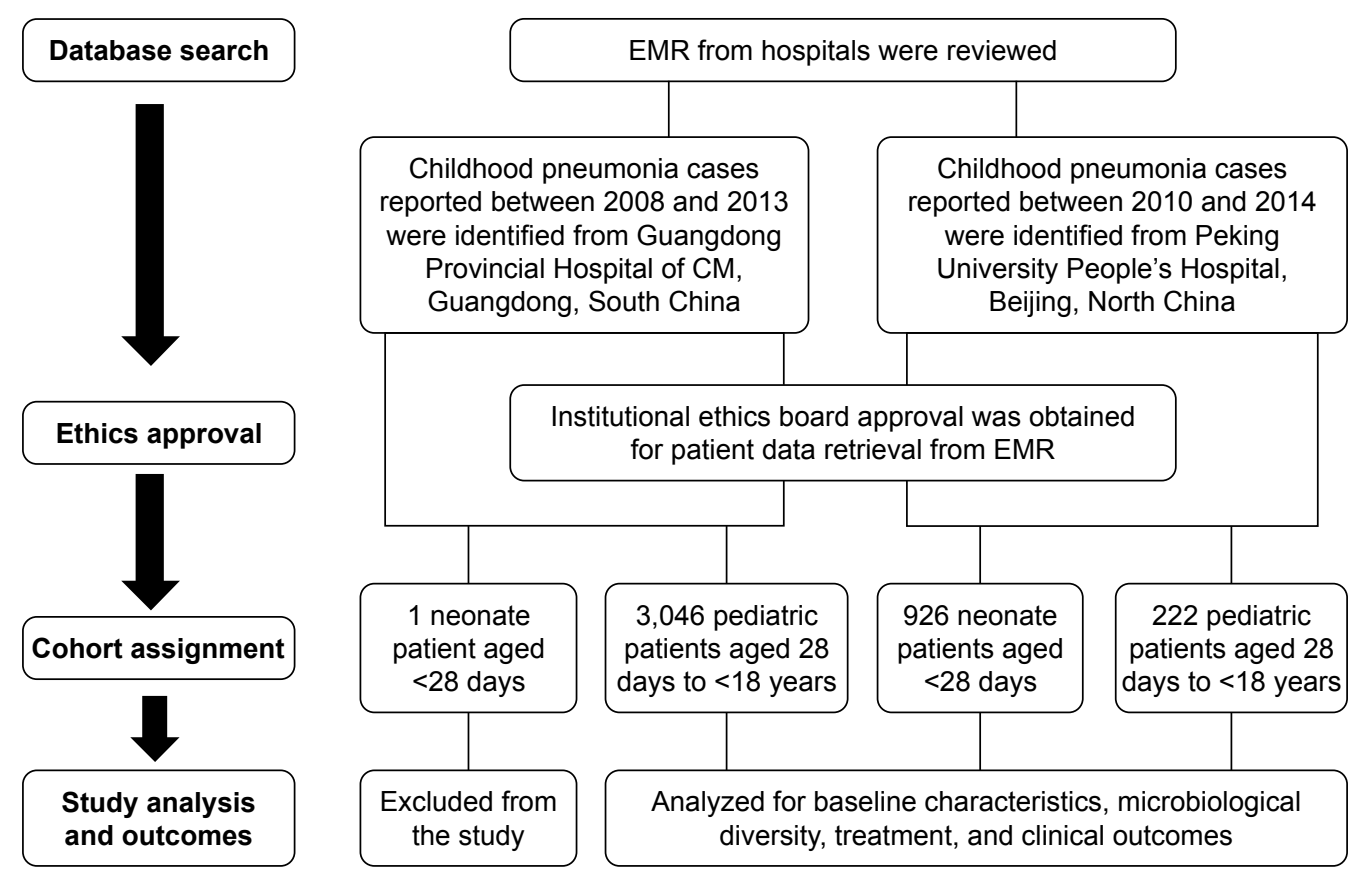

Figure I Study flow chart.

Abbreviations: CM, Chinese Medicine; EMR, electronic medical records.

Hospitalizations receiving CM only (without any conventional antimycotic, antiviral, or antibiotic therapy) were excluded from the clinical outcome analyses to avoid biasing the results.

\section{Ethical considerations}

Individual patient data were anonymized, and ethical approval was granted by the relevant institutional ethics board committees at each site (Ethics Committee of Guangdong Provincial Hospital of CM and Ethics Committee of Peking University People's Hospital). This study involved the collection of existing data and records. Informed consent was exempted according to the decision of institutional ethics board committees.

\section{Data collection}

Study variables included patient baseline demographics, medical history (including comorbidities and surgical intervention), frequently used antibiotics, and clinical outcomes. Demographic information extracted for analysis included: age, gender and ethnic origin. Clinical outcomes data extracted for analysis included length of hospital stay, recurrence of infection, ICU admission, discharge from hospital, and in-hospital mortality. Microbiological data regarding bacteria identified by culture tests (except for M. pneumoniae in Beijing, which was identified using serological methods) of patient sputum/respiratory secretions and blood samples, which were collected when possible/as clinically indicated, were also extracted. Bacteria were identified according to the Clinical and Laboratory Standards Institute guidelines. ${ }^{15}$

\section{Statistical analysis}

All descriptive analyses were conducted using R Statistics Software (Version R3.1.1). Continuous data are expressed as the mean \pm standard deviation. Categorical data are expressed as the number of events and percentages.

\section{Results \\ Baseline characteristics}

The study included 3,046 pediatric hospitalizations $(2,706$ unique patients) from Guangdong Provincial Hospital and 926 neonatal and 226 pediatric hospitalizations $(1,112$ unique patients) from Peking University People's Hospital. One patient may have more than one hospitalization visit. Since only one neonatal hospitalization was identified from Guangdong Provincial Hospital of CM, it was excluded from the study. The most common comorbidities were hospital tonsillitis, bronchial disorder, and anemia in Guangdong pediatric hospitalizations, whereas blood tumor was predominant in pediatric hospitalizations of Peking University People's Hospital. Neonatal hospitalizations of Peking University People's Hospital presented anemia, neonatal hyperalbuminemia, and septicemia. Further details on baseline demographics, concomitant medication use, and surgical intervention are presented in Table 1. 
Table I Baseline patient demographics, comorbidities, and surgical interventions

\begin{tabular}{|c|c|c|c|}
\hline \multirow[t]{2}{*}{ Baseline characteristics } & \multicolumn{2}{|c|}{ Peking University People's Hospital } & \multirow{2}{*}{$\begin{array}{l}\text { Guangdong Provincial } \\
\text { Hospital of CM } \\
28 \text { days to I7 years } \\
(n=3,046)\end{array}$} \\
\hline & $\begin{array}{l}0-27 \text { days } \\
(n=926)\end{array}$ & $\begin{array}{l}28 \text { days to } 17 \text { years } \\
(n=222)\end{array}$ & \\
\hline Age (years), mean \pm SD (median) & $3.5 \pm 5.9(0)$ & $6.5 \pm 5.6(5)$ & $3.3 \pm 3.1(2)$ \\
\hline \multicolumn{4}{|l|}{ Gender, n (\%) } \\
\hline Male & $526(56.8)$ & $130(58.6)$ & $\mathrm{I}, 793(58.8)$ \\
\hline Female & $400(43.2)$ & $92(4 \mid .4)$ & $\mathrm{I}, 253(4 \mathrm{I} . \mathrm{I})$ \\
\hline \multicolumn{4}{|l|}{ Ethnic origin, $\mathrm{n}(\%)$} \\
\hline Han Chinese & $895(96.7)$ & $188(84.7)$ & $3,027(99.4)$ \\
\hline Non-Han Chinese & $29(3.1)$ & $10(4.5)$ & $16(0.5)$ \\
\hline Unknown & $2(0.2)$ & $24(10.8)$ & $3(0.1)$ \\
\hline Comorbidities, n (\%) & $921(99.5)$ & $208(93.7)$ & $3,046(100.0)$ \\
\hline \multicolumn{4}{|l|}{ Respiratory disease } \\
\hline Bronchial disorder & $0(0.0)$ & $0(0.0)$ & $66(2.2)$ \\
\hline Bronchopulmonary dysplasia & $16(1.7)$ & $\mathrm{I}(0.5)$ & $0(0.0)$ \\
\hline Tuberculosis & $0(0.0)$ & $0(0.0)$ & $2(0.1)$ \\
\hline Other lung infections & $0(0.0)$ & $3(1.4)$ & $0(0.0)$ \\
\hline Tonsillitis & $0(0.0)$ & $0(0.0)$ & $114(3.7)$ \\
\hline Anemia & $497(53.7)$ & $29(13.1)$ & $38(1.3)$ \\
\hline \multicolumn{4}{|l|}{ Malignant tumor } \\
\hline Solid tumor & $0(0.0)$ & $\mathrm{I}(0.5)$ & $\mathrm{I}(0.0)$ \\
\hline Blood tumor & $2(0.2)$ & $135(60.8)$ & $0(0.0)$ \\
\hline Neonatal hyperbilirubinemia & $318(34.3)$ & $2(0.9)$ & $0(0.0)$ \\
\hline Septicemia & $54(5.8)$ & $18(8.1)$ & $14(0.5)$ \\
\hline Renal failure & $0(0.0)$ & $0(0.0)$ & $\mathrm{I}(0.0)$ \\
\hline \multicolumn{4}{|l|}{ Treatment and medication, $\mathrm{n}(\%)$} \\
\hline Surgical treatment in the & $0(0.0)$ & $0(0.0)$ & $3(0.1)$ \\
\hline \multicolumn{4}{|l|}{3 months prior to hospitalization } \\
\hline Medication in the 3 months & $0(0.0)$ & $15(6.8)$ & $62(2.0)$ \\
\hline prior to hospitalization & & & \\
\hline
\end{tabular}

Abbreviation: CM, Chinese Medicine.

\section{Pathogen profiling}

Microbiological characteristics and outcomes of pediatric hospitalizations in Guangdong Provincial Hospital of CM

A total of 241 microbiological samples were culture positive, the majority (180/241 [74.7\%]) of which were collected from pharyngeal swabs. Fifty $(20.7 \%)$ culture positive samples were collected from sputum, nine $(3.7 \%)$ samples were collected from blood, and two $(0.8 \%)$ samples were collected from BAL. Bacteria were isolated from 236/3,046 (7.7\%) hospitalizations; 21 (8.9\%) had multiple bacterial organisms identified, and the remainder had a single bacterial organism identified. Two hundred seven (78.1\%) of the 265 bacteria isolated were from samples collected within 2 days of hospital admission. The atypical organism M. pneumoniae was the most frequently isolated organism, identified in 154/236 (65.3\%) hospitalizations (Table 2). The most common bacteria were $S$. aureus (20/236 [8.5\%]) and Acinetobacter baumanii (12/236 [5.1\%]; Table 2). Of the 20 hospitalizations in whom $S$. aureus was isolated, three had MRSA and 17 had methicillin-susceptible S. aureus (MSSA).

\section{Microbiological characteristics and outcomes of neonatal hospitalizations in Peking University People's Hospital}

A total of 25 microbiological samples were culture positive, of which 24 samples were collected from blood and one sample was obtained from sputum. Bacteria were isolated from 24/926 (2.6\%) neonates; one neonate had two bacterial organisms identified ( $P$. aeruginosa + Listeria monocytogenes), and the remainder had a single bacterial organism identified. Twenty-one (84.0\%) of the 25 bacteria isolated were from samples collected within 2 days of hospital admission. The most common bacteria, isolated in 10/24 (41.7\%) hospitalizations, was S. epidermidis (Table 2). 
Table 2 Bacteria isolated $\geq 2$ hospitalizations at either hospital ${ }^{\mathrm{a}}$

\begin{tabular}{|c|c|c|c|}
\hline \multirow[t]{2}{*}{ Bacterial strains } & \multicolumn{2}{|c|}{ Peking University People's Hospital } & \multirow{2}{*}{$\begin{array}{l}\text { Guangdong Provincial } \\
\text { Hospital of } \mathrm{CM} \\
28 \text { days to } 17 \text { years }\end{array}$} \\
\hline & $0-27$ days & 28 days to 17 years & \\
\hline Hospitalizations with a & $24(2.6)$ & $14(6.3)$ & $236(7.7)$ \\
\hline \multicolumn{4}{|l|}{ microbiological diagnosis, n (\%) } \\
\hline \multicolumn{4}{|l|}{ Gram-negative bacteria, n (\%) } \\
\hline \multicolumn{4}{|l|}{ Enterobacteriaceae } \\
\hline Klebsiella pneumoniae & $2(8.3)$ & $0(0.0)$ & $8(3.4)$ \\
\hline Escherichia coli & I (4.2) & $0(0.0)$ & $9(3.8)$ \\
\hline Enterobacter cloacae & I (4.2) & $0(0.0)$ & $6(2.5)$ \\
\hline \multicolumn{4}{|l|}{ Pseudomonas spp. } \\
\hline Pseudomonas aeruginosa & I (4.2) & I (7.I) & $6(2.5)$ \\
\hline \multicolumn{4}{|l|}{ Moraxella spp. } \\
\hline Acinetobacter junii & $0(0.0)$ & $3(2 \mid .4)$ & $0(0.0)$ \\
\hline Acinetobacter baumannii & $0(0.0)$ & I (7.I) & $12(5.1)$ \\
\hline Branhemella catarrhalis & $0(0.0)$ & $0(0.0)$ & $7(3.0)$ \\
\hline \multicolumn{4}{|l|}{ Xanthomonas spp. } \\
\hline \multicolumn{4}{|l|}{ Haemophilus spp. } \\
\hline Haemophilus influenzae & $0(0.0)$ & $0(0.0)$ & $8(3.4)$ \\
\hline \multicolumn{4}{|l|}{ Gram-positive bacteria, n (\%) } \\
\hline \multicolumn{4}{|l|}{ Staphylococcus spp. } \\
\hline Staphylococcus aureus & $0(0.0)$ & $0(0.0)$ & $20(8.5)$ \\
\hline Methicillin-resistant S. aureus & $0(0.0)$ & $0(0.0)$ & $3(1.3)$ \\
\hline Methicillin-susceptible S. aureus & $0(0.0)$ & $0(0.0)$ & $17(7.2)$ \\
\hline Staphylococcus epidermidis & $10(41.7)$ & $\mathrm{I}(7.1)$ & I $(0.4)$ \\
\hline Staphylococcus haemolyticus & $4(16.7)$ & $0(0.0)$ & $0(0.0)$ \\
\hline Staphylococcus capitis & $2(8.3)$ & $0(0.0)$ & $0(0.0)$ \\
\hline Staphylococcus hominis & $\mathrm{I}(4.2)$ & I (7.I) & $3(1.3)$ \\
\hline \multicolumn{4}{|l|}{ Streptococcus spp. } \\
\hline Streptococcus pneumoniae & $0(0.0)$ & $0(0.0)$ & $7(3.0)$ \\
\hline \multicolumn{4}{|l|}{ Atypical organisms, n (\%) } \\
\hline \multicolumn{4}{|l|}{ Mycoplasma spp. } \\
\hline Mycoplasma pneumoniae & $0(0.0)$ & $7 / 50(14.0)^{\mathrm{b}}$ & $154(65.3)$ \\
\hline
\end{tabular}

Notes: aSome pathogens were identified in multiple different samples from a single patient. 'blentified from blood sample by antibody. Abbreviation: CM, Chinese Medicine.

\section{Microbiological characteristics and outcomes of pediatric hospitalizations in Peking University People's Hospital}

A total of 14 microbiological samples were culture positive, of which 7/14 (50.0\%) were collected from sputum and 7/14 $(50.0 \%)$ were obtained from blood; none were obtained from BAL, pharyngeal swabs, or bronchial secretions. Eight of the 18 (44.4\%) bacteria isolated were from samples collected within 2 days of hospital admission. Bacteria were isolated from 14/222 (6.3\%) hospitalizations; a single bacterial organism was identified in 11 hospitalizations; multiple bacterial organisms were identified in the remaining three hospitalizations. The bacteria most frequently identified using culture-based methods was Acinetobacter junii, isolated in $3 / 14$ (21.4\%) hospitalizations (Table 2). Of the 50 hospitalizations, whose blood was tested for antibodies against M. pneumoniae, seven (14.0\%) were positive.

\section{Choice of treatment and outcomes Clinical outcomes and treatment patterns of pediatric hospitalizations in Guangdong Provincial Hospital of CM}

The mean (SD) length of hospital stay was 5.8 (3) days, and the median length of stay was 5 days. The majority of hospitalizations $(2,526 / 3,046$ [82.9\%]) received antibiotic therapy; among these hospitalizations, medication was modified in 625 (24.7\%) hospitalizations (Figure 2).

In addition, CM was used only in Guangdong Provincial Hospital of CM. In total, CM was used in 2,814/3,046 (92.4\%) hospitalizations. Four hundred fifty-two (14.9\%) hospitalizations received CM without any concomitant antimycotic, antiviral, or antibiotic therapy and were excluded from the clinical outcome analyses. Clinical outcomes for the remaining 2,545/3,046 (83.6\%) hospitalizations who received conventional antimycotic, antiviral, or antibiotic 

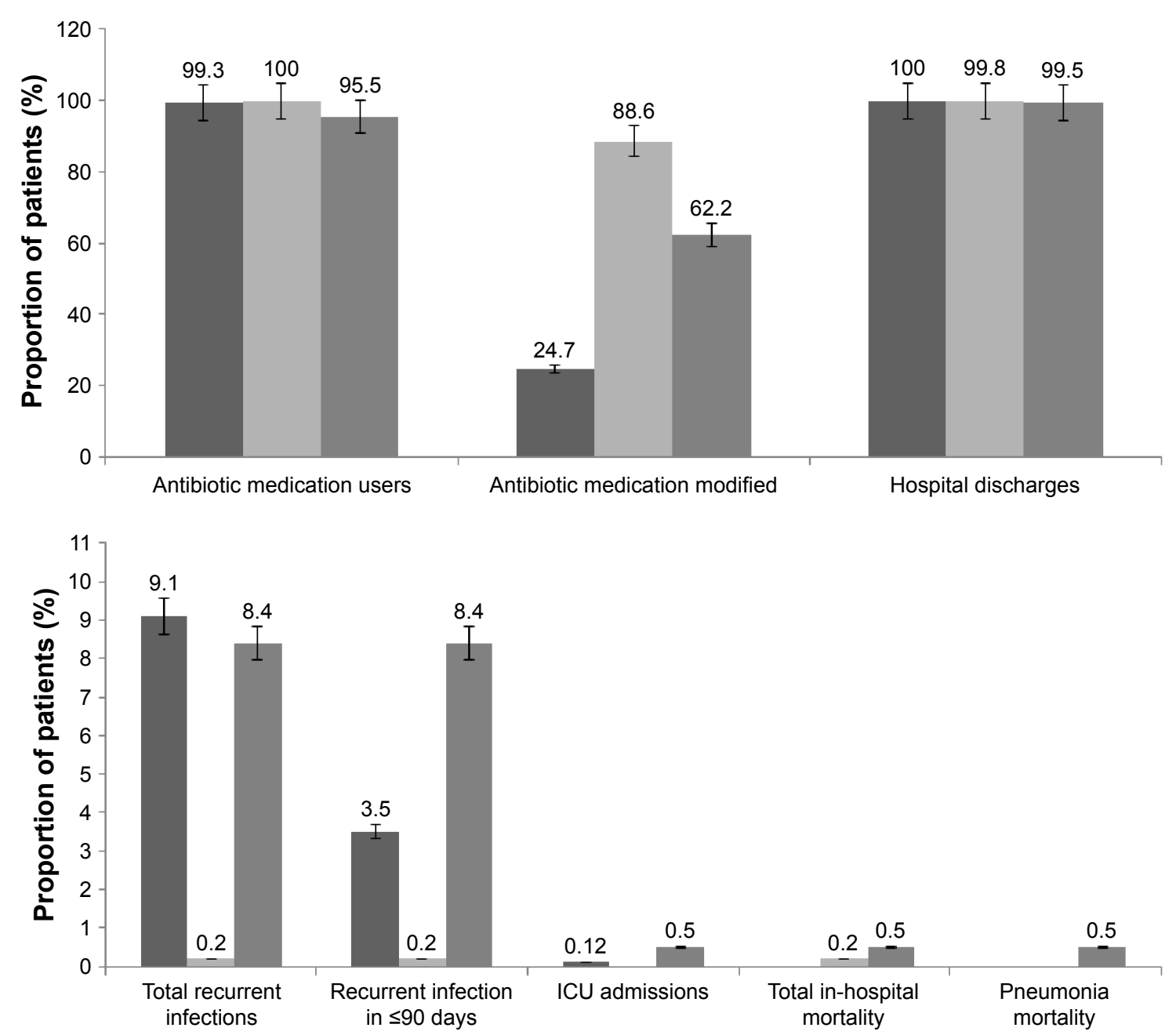

Figure 2 Clinical outcomes of neonatal and pediatric patients in Guangdong and Peking University hospitals. Abbreviation: ICU, intensive care unit.

therapy are detailed in Table 3. The mean (SD) length of hospital stay was 5.8 (3.0) days, and the median length of stay was 5 days. Recurrence of infection within 90 days was low $(80 / 2,297$ [3.5\%] patients for whom recurrence data were available). The majority of hospitalized patients were discharged, only one patient reported death caused by severe influenza A (H1N1) virus infection, which was not considered attributable to pneumonia infection.

As $S$. aureus was the most common bacteria isolated, clinical outcomes of hospitalizations with $S$. aureus infection were assessed. The mean length of stay was similar in hospitalizations with MRSA (6.0 days) and longer in hospitalizations with MSSA (9.4 days) when compared with the overall population (5.7 days). None of the hospitalizations with MRSA or MSSA died due to pneumonia. Note that there were only three hospitalizations with MRSA (Table 2); due to the low number there may be a bias in the results.

\section{Clinical outcomes and treatment patterns of neonatal hospitalizations in Peking University People's Hospital}

The mean (SD) length of hospital stay was 11.2 (7.4) days, and the median length of stay was 9 days. The majority of hospitalized patients were discharged (924/926 [99.8\%]), while two patients died during the hospitalization course, and these deaths were not attributable to pneumonia infection. Recurrence of infection within 90 days was low (2/924 patients for whom recurrence data were available). Of all the patients given antibiotics, modification of initial antibiotic treatment occurred in 820/926 (88.6\%) patients (Figure 2).

\section{Clinical outcomes and treatment patterns of pediatric hospitalizations in Peking University People's Hospital}

The mean (SD) length of hospital stay was 17.4 (19.9) days, and the median length of stay was 11 days. The majority of 
Table 3 Frequently used antibiotics for initial therapy and overall at both the study hospitals

\begin{tabular}{|c|c|c|c|c|c|c|}
\hline \multirow[t]{4}{*}{ Antibiotics } & \multicolumn{6}{|c|}{ Number of patients receiving antibiotics during hospitalization, $\mathbf{n}(\%)$} \\
\hline & \multirow{2}{*}{\multicolumn{2}{|c|}{$\begin{array}{l}\text { Guangdong Provincial } \\
\text { Hospital of CM (age } 28 \text { days } \\
\text { to I } 7 \text { years, } n=2,526 \text { ) }\end{array}$}} & \multirow{2}{*}{\multicolumn{2}{|c|}{$\begin{array}{l}\text { Peking University } \\
\text { People's Hospital (age } \\
\text { 0-27 days, } n=926 \text { ) }\end{array}$}} & \multirow{2}{*}{\multicolumn{2}{|c|}{$\begin{array}{l}\text { Peking University People's } \\
\text { Hospital (age } 28 \text { days to } \\
\text { I } 7 \text { years, } n=212 \text { ) }\end{array}$}} \\
\hline & & & & & & \\
\hline & & Overall & Initial & Oyerall & Initial & Oyerall \\
\hline \multicolumn{7}{|l|}{ Beta-lactam antibacterials } \\
\hline Penicillins & $147(5.82)$ & $195(7.72)$ & $19(77.7)$ & $799(86.3)$ & $28(\mid 3.2)$ & $46(21.7)$ \\
\hline$\beta$-Lactamase sensitive penicillins & $|4|(5.58)$ & $180(7.13)$ & $717(77.4)$ & $797(86.1)$ & $25(I 1.8)$ & $39(18.4)$ \\
\hline Benzylpenicillin & $|4|(5.58)$ & $180(7.13)$ & $717(77.4)$ & $797(86.1)$ & $25(I 1.8)$ & $39(18.4)$ \\
\hline Combinations of penicillins, including & $104(4.12)$ & $140(5.54)$ & $2(0.2)$ & $2(0.2)$ & $\mathrm{I}(0.5)$ & $8(3.8)$ \\
\hline \multicolumn{7}{|l|}{$\beta$-lactamase inhibitors } \\
\hline Amoxicillin and clavulanic acid & $101(4.00)$ & $133(5.27)$ & $2(0.2)$ & $2(0.2)$ & $0(0.00)$ & $0(0.00)$ \\
\hline Piperacillin and tazobactam & $0(0.00)$ & $0(0.00)$ & $0(0.00)$ & $0(0.00)$ & $\mathrm{I}(0.5)$ & $8(3.8)$ \\
\hline Penicillins with extended spectrum & $0(0.00)$ & $22(0.87)$ & $0(0.00)$ & $0(0.00)$ & $4(1.9)$ & II (5.2) \\
\hline Amoxicillin & $0(0.00)$ & $22(0.87)$ & $0(0.00)$ & $0(0.00)$ & $0(0.00)$ & $0(0.00)$ \\
\hline Piperacillin & $0(0.00)$ & $0(0.00)$ & $0(0.00)$ & $0(0.00)$ & $4(1.9)$ & $10(4.7)$ \\
\hline Carbapenems & $19(0.75)$ & $7 \mathrm{I}(2.8 \mathrm{I})$ & $\mathrm{I}(0.1)$ & $37(4.0)$ & $50(23.6)$ & $97(45.8)$ \\
\hline Meropenem & $0(0.00)$ & $0(0.00)$ & $\mathrm{I}(0.1)$ & $37(4.0)$ & $50(23.6)$ & $97(45.8)$ \\
\hline Imipenem-cilastatin & $6(0.24)$ & $29(1.15)$ & $0(0.00)$ & $0(0.00)$ & $0(0.00)$ & $0(0.00)$ \\
\hline First-generation cephalosporins & $5(0.20)$ & $7(0.28)$ & $0(0.00)$ & $0(0.00)$ & $0(0.00)$ & $0(0.00)$ \\
\hline Cefathiamidine & $2(0.08)$ & $3(0.12)$ & $0(0.00)$ & $0(0.00)$ & $0(0.00)$ & $0(0.00)$ \\
\hline Second-generation cephalosporins & $188(7.44)$ & $221(8.75)$ & $0(0.00)$ & $0(0.00)$ & $20(9.4)$ & $41(19.3)$ \\
\hline Cefuroxime & $162(6.4 \mathrm{I})$ & $175(6.93)$ & $0(0.00)$ & $0(0.00)$ & $17(8.0)$ & $24(11.3)$ \\
\hline Cefaclor & $15(0.59)$ & $32(1.27)$ & $0(0.00)$ & $0(0.00)$ & $4(1.9)$ & $21(9.9)$ \\
\hline Third-generation cephalosporins & I,07I (42.40) & $\mathrm{I}, 238(49.0 \mathrm{I})$ & $391(42.2)$ & $586(63.3)$ & $74(34.9)$ & $118(55.7)$ \\
\hline Ceftriaxone & $996(39.43)$ & $\mathrm{I}, \mathrm{I} 30(44.73)$ & $0(0.00)$ & $2(0.2)$ & $9(4.3)$ & II (5.2) \\
\hline Cefixime & $34(1.35)$ & $299(11.84)$ & $0(0.00)$ & $0(0.00)$ & $5(2.4)$ & $41(19.3)$ \\
\hline Ceftazidime & $0(0.00)$ & $0(0.00)$ & $391(42.2)$ & $586(63.3)$ & $56(26.4)$ & $80(37.7)$ \\
\hline Cefoperazone and sulbactam & $0(0.00)$ & $0(0.00)$ & $0(0.00)$ & $0(0.00)$ & $5(2.4)$ & $7(3.3)$ \\
\hline Fourth-generation cephalosporins & $0(0.00)$ & $0(0.00)$ & $33(3.6)$ & $179(19.3)$ & $9(4.3)$ & $33(15.6)$ \\
\hline Cefepime & $0(0.00)$ & $0(0.00)$ & $33(3.6)$ & $179(19.3)$ & $9(4.3)$ & $33(15.6)$ \\
\hline Macrolides, lincosamides, and streptogramins & $\mathrm{I}, 273(50.40)$ & $1,735(68.69)$ & $13(1.4)$ & $773(83.5)$ & $60(28.3)$ & $138(65.1)$ \\
\hline Macrolides & I, 199 (47.47) & $\mathrm{I}, 685(66.7 \mathrm{I})$ & $13(1.4)$ & $773(83.5)$ & $60(28.3)$ & $137(64.6)$ \\
\hline Azithromycin & $\mathrm{I}, \mathrm{I} 52(45.6 \mathrm{I})$ & $\mathrm{I}, 655(65.52)$ & $13(1.4)$ & $768(82.9)$ & $59(27.8)$ & $133(62.7)$ \\
\hline Erythromycin & $42(1.66)$ & $80(3.17)$ & $0(0.00)$ & $8(0.9)$ & $\mathrm{I}(0.5)$ & $9(4.2)$ \\
\hline Lincosamides & $75(2.97)$ & $89(3.52)$ & $0(0.00)$ & $0(0.00)$ & $0(0.00)$ & $0(0.00)$ \\
\hline Clindamycin & $75(2.97)$ & $89(3.52)$ & $0(0.00)$ & $0(0.00)$ & $0(0.00)$ & $0(0.00)$ \\
\hline Aminoglycoside antibacterials & I (0.04) & $4(0.16)$ & $14(1.5)$ & $99(10.7)$ & $13(6.1)$ & $30(14.2)$ \\
\hline Gentamicin & I (0.04) & $3(0.12)$ & & & $9(4.3)$ & $18(8.5)$ \\
\hline Tobramycin & $0(0.00)$ & $0(0.00)$ & $14(1.5)$ & $99(10.7)$ & $4(1.9)$ & $10(4.7)$ \\
\hline Amphenicols & $0(0.00)$ & $0(0.00)$ & $0(0.00)$ & $0(0.00)$ & $7(3.3)$ & $7(3.3)$ \\
\hline Chloramphenicol & $0(0.00)$ & $0(0.00)$ & $0(0.00)$ & $0(0.00)$ & $7(3.3)$ & $7(3.3)$ \\
\hline Sulfonamides and trimethoprim & $0(0.00)$ & $0(0.00)$ & $0(0.00)$ & $0(0.00)$ & $5(2.4)$ & $5(2.4)$ \\
\hline Intermediate-acting sulfonamides & $0(0.00)$ & $0(0.00)$ & $0(0.00)$ & $0(0.00)$ & $5(2.4)$ & $5(2.4)$ \\
\hline Sulfamethoxazole & $0(0.00)$ & $0(0.00)$ & $0(0.00)$ & $0(0.00)$ & $5(2.4)$ & $5(2.4)$ \\
\hline Quinolone antibacterials & $0(0.00)$ & $0(0.00)$ & $0(0.00)$ & $0(0.00)$ & $4(1.9)$ & $16(7.6)$ \\
\hline Fluoroquinolones & $0(0.00)$ & $0(0.00)$ & $0(0.00)$ & $0(0.00)$ & $4(1.9)$ & $16(7.6)$ \\
\hline Norfloxacin & $0(0.00)$ & $0(0.00)$ & $0(0.00)$ & $0(0.00)$ & $2(0.9)$ & $2(0.9)$ \\
\hline Levofloxacin & $0(0.00)$ & $0(0.00)$ & $0(0.00)$ & $0(0.00)$ & $0(0.00)$ & $8(3.8)$ \\
\hline Other antibacterials & $3(0.12)$ & $5(0.20)$ & $0(0.00)$ & $0(0.00)$ & $53(25.0)$ & $108(50.9)$ \\
\hline Steroid antibacterials & $\mathrm{I}(0.04)$ & $2(0.08)$ & $0(0.00)$ & $0(0.00)$ & $0(0.00)$ & $0(0.00)$ \\
\hline Fusidic acid & I (0.04) & $2(0.08)$ & $0(0.00)$ & $0(0.00)$ & $0(0.00)$ & $0(0.00)$ \\
\hline Glycopeptide antibacterials & $0(0.00)$ & $0(0.00)$ & $0(0.00)$ & $0(0.00)$ & $47(22.2)$ & $102(48.1)$ \\
\hline Norvancomycin & $0(0.00)$ & $0(0.00)$ & $0(0.00)$ & $0(0.00)$ & $47(22.2)$ & $96(45.3)$ \\
\hline Vancomycin & $0(0.00)$ & $0(0.00)$ & $0(0.00)$ & $0(0.00)$ & $0(0.00)$ & $12(5.7)$ \\
\hline Teicoplanin & $0(0.00)$ & $0(0.00)$ & $0(0.00)$ & $0(0.00)$ & $0(0.00)$ & $7(3.3)$ \\
\hline Imidazole derivatives & $0(0.00)$ & $0(0.00)$ & $0(0.00)$ & $0(0.00)$ & $18(8.5)$ & $102(48.1)$ \\
\hline Metronidazole & $0(0.00)$ & $0(0.00)$ & $0(0.00)$ & $0(0.00)$ & $18(8.5)$ & $35(16.5)$ \\
\hline Others & I (0.04) & $4(0.16)$ & $0(0.00)$ & $0(0.00)$ & $0(0.00)$ & $12(5.7)$ \\
\hline Linezolid & I $(0.04)$ & $4(0.16)$ & $0(0.00)$ & $0(0.00)$ & $0(0.00)$ & $12(5.7)$ \\
\hline
\end{tabular}

Abbreviation: CM, Chinese Medicine. 
hospitalizations (221/222 [99.5\%]) were discharged. One hospitalized patient died, and the death was considered attributable to the pneumonia infection. Recurrence of infection within 90 days was low (16/190 [8.4\%] patients for whom recurrence data were available). The majority of hospitalizations (212/222 [95.5\%]) received antibiotic therapy; among these hospitalizations, medication was modified in $138(62.2 \%)$ patients (Figure 2). The most frequently used antibiotics at both hospitals for neonatal and pediatric patients are listed in Table 3.

\section{Discussion}

This retrospective, observational study gathered detailed epidemiological information on patient characteristics, treatment patterns, and clinical outcomes in neonatal and pediatric pneumonia hospitalizations from hospitals in different regions of China. It is plausible that, as in Western countries, there is a high frequency of viral pneumonia in pediatric populations in China, ${ }^{6}$ potentially explaining the low isolation rate of bacteria. The most common bacterial organism isolated in pediatric hospitalizations in Beijing was A. junii. Acinetobacter species are commonly found in the context of HAP, ${ }^{16}$ and it is plausible that $A$. junii infection may have been acquired in the hospital setting due to the long mean length of hospital stay observed in pediatric hospitalizations in Beijing. However, due to the low number of hospitalizations with at least one bacterial organism isolated and the fact that it was impossible to distinguish between colonization and infection in this study, clearly determining the microbiological cause of pneumonia in these hospitalizations was not possible.

M. pneumoniae was the predominant bacteria isolated from pediatric hospitalizations in Guangdong, followed by S. aureus and A. baumanii. M. pneumoniae is a well-known cause of CAP among pediatric patients, ${ }^{17}$ and a high prevalence of atypical pathogens among Asian patients with CAP has been reported in a previous study. ${ }^{17}$ There was a greater prevalence of M. pneumoniae in Guangdong than in Beijing. However, it should be noted that different detection methods were used for M. pneumoniae at the two sites - culture was used in Guangdong and antibody testing in Beijing - highlighting the difficulty in the comparison of retrospective data from two independent study centers. There was a low level of in-hospital mortality due to pneumonia (zero in Guangdong and close to zero in Beijing), with 99.6\%-100\% of hospitalizations being discharged. The high proportion of M. pneumoniae isolates may be a factor in this low mortality rate, as $M$. pneumoniae is generally associated with favorable clinical outcomes. ${ }^{18}$ Additionally, previous studies indicate that the most fatal pneumonia cases in children are caused by H. influenzae or S. pneumoniae $;^{19}$ the low level of isolation of these bacteria in the current study may help to explain the low number of deaths due to pneumonia. The mean length of stay was considerably longer for pediatric hospitalizations in Beijing as compared with Guangdong, and the frequency of modification of antibiotic therapy was three times higher. The fact that Beijing is a tertiary referral facility for patients requiring specialist care might explain this finding, as it is plausible that there may have been a greater proportion of severe or complicated cases compared with Guangdong Provincial Hospital of CM. Recurrence of infection was rare in neonatal hospitalizations $(0.2 \%)$, and in pediatric hospitalizations, the frequency of recurrence was the same across the participating hospitals $(8.4 \%$ in both Beijing and Guangdong). The frequency of ICU admission was close to zero at both sites in all patient subgroups. In hospitalizations with $S$. aureus infection, the length of stay was longer and the frequency of antibiotic modification was higher compared with the overall population; however, no deaths occurred in hospitalizations with $S$. aureus infection. The causative pathogens implicated in pediatric pneumonia are distinct in developed countries where there is greater involvement of viral and atypical organisms $\mathrm{s}^{20-23}$ compared with developing countries where bacterial pathogens account for a significant proportion (60\%) of pneumonia cases, ${ }^{24}$ the most important of which are S. pneumoniae and H. influenza. ${ }^{17,25}$ Pneumococcal vaccination is not common in China and, therefore, cannot explain the low frequency of $S$. pneumoniae isolated in the current study. Pneumococcal urinary antigen tests were not performed at either of the hospitals; this is in line with Western guidelines, which recommend that urinary pneumococcal antigen detection should not be done in young children. ${ }^{11,26}$ S. epidermidis was the most common bacteria isolated in neonatal hospitalizations in Beijing. Given that all the 10 isolates were from blood samples, it is possible that this is an artifact of contamination with skin flora. ${ }^{27}$ However, S. epidermidis has been linked to neonatal pneumonia of late onset ( $>48$ hours after birth) and pneumonia in immunocompromised individuals ${ }^{28,29}$ and is a recognized HAP pathogen in pediatric patients. S. epidermidis was recently identified as a leading opportunistic pathogen in neonatal sepsis at another hospital in China, ${ }^{30}$ and in the current study, nine of the 10 neonatal hospitalizations with $S$. epidermidis isolated from blood cultures had comorbid sepsis. It is possible that these bacteria may have been associated with the comorbid septicemia that was reported in $5.9 \%$ of neonatal hospitalizations in Beijing, rather than pneumonia. The low level of positive bacterial cultures and the high levels of 
modification of initial antibiotic in the present study imply that empirical antibiotic therapy was generally modified on the basis of clinical signs. These data highlight the difficulties faced in providing appropriate empiric therapy, which must ultimately be guided by recent knowledge of local pathogens and resistance patterns and is limited in the current study by the low bacterial isolation rates. In the present study, the combination of a macrolide with a cephalosporin was the most common initial therapy. However, it should be noted that in patients for whom bacteria could be isolated, initial antibiotic coverage was de-escalated based upon patientspecific culture results.

The limitation that must be taken into consideration when comparing findings between the Guangdong and Beijing hospitals is the differing patient age distributions between the hospitals. Only one neonatal hospitalization was identified with pneumonia in Guangdong Provincial Hospital of $\mathrm{CM}$ during the study period, likely as a result of its small maternity department, and therefore, data of neonates from this site were excluded from this analysis. Furthermore, for the purposes of this study, pediatric and adolescent hospitalizations aged 28 days to $<18$ years were grouped into one category, which is a wide age range from an epidemiological point of view. In Guangdong, most hospitalizations were aged 3 weeks to 5 years, whereas the majority of hospitalizations in Beijing were neonatal hospitalizations (aged 0-27 days). Etiological agents commonly responsible for pneumonia differ by age group; ${ }^{6}$ therefore, this variation must be taken into consideration. A further limitation of this study is that it was not possible to distinguish between CAP and HAP.

However, it is rare for EMR to be used for retrospective studies in Asia, especially in China. ${ }^{31}$ EMR system is still relatively new in China compared to western countries, and the difficulty in cleaning data from Chinese EMR system is also a big problem. In addition, it is hard to access the EMR systems from different hospitals due to the lack of readiness to share data. Our work is the first study in China to compare microbial profile and current treatment practices between two large conglomerate hospitals, which may reflect the scenario and thrust areas of improvements in the field of pneumonia.

\section{Conclusion}

This large, retrospective observational study of hospitalizations in different regions of China provides new epidemiological insights into neonatal and pediatric pneumonia in China. The findings revealed M. pneumoniae and $S$. epidermis as the most common pathogen found in children of Guangdong and Beijing, respectively. There was a low level of in-hospital mortality due to pneumonia, and the majority of hospitalizations were discharged from hospital, suggesting that current practice was generally effective; however, guidelines recommend obtaining microbiological diagnoses wherever possible. We also found that neonatal hospitalizations were greater than pediatric hospitalizations in Beijing, proposing a need to improve neonatal pneumonia prophylaxis and selection of appropriate treatment. In addition, ongoing local and regional surveillance is necessary to monitor the antimicrobial susceptibility and prevalence of pathogens associated with the infection and prescribing patterns.

\section{Acknowledgments}

This study was funded by AstraZeneca. Medical writing support was provided by Liz Bolton of Prime Medica Ltd, Knutsford, Cheshire, UK, and Dr Amit Bhat of Indegene Pvt Ltd, Bangalore, India, funded by AstraZeneca. The authors would like to acknowledge Weiguo Gao of AstraZeneca R\&D Information, China, for the medical advice, Shizhou Deng of Peking University People's Hospital for the data collection support, and Tong Zhu of Yidu Cloud Technology Co., Ltd, for the extraction, integration, and linking of data from EMR. This helped to clarify certain statistically relevant epidemiological characteristics of pediatric pneumonia, which was considered valuable to the subsequent research. The design and conduct of the study, as well as analysis of the study data and opinions, conclusions, and interpretation of the data, are the responsibility of the authors.

\section{Author contributions}

ZW, JW, and HX conceptualized the study. All authors contributed toward data analysis, drafting and critically revising the paper, gave final approval of the version to be published, and agree to be accountable for all aspects of the work. ZW and JW contributed equally to this work.

\section{Disclosure}

JW, HX, and JH are employees of AstraZeneca. DM and JG are former employees of AstraZeneca. ZW, XL, and $\mathrm{ZC}$ received institutional/research grant funding from AstraZeneca for the conduct of the study. The authors report no other conflicts of interest in this work.

\section{References}

1. Rudan I, Boschi-Pinto C, Biloglav Z, Mulholland K, Campbell H. Epidemiology and etiology of childhood pneumonia. Bull World Health Organ. 2008;86(5):408-416.

2. Guan X, Silk BJ, Li W, et al. Pneumonia incidence and mortality in mainland China: systematic review of Chinese and English literature, 1985-2008. PLoS One. 2010;5(7):e11721. 
3. Duke T. Neonatal pneumonia in developing countries. Arch Dis Child Fetal Neonatal Ed. 2005;90(3):F211-F219.

4. Nissen MD. Congenital and neonatal pneumonia. Paediatr Respir Rev. 2007;8(3):195-203.

5. Martins AL, Nascimento DD, Schneider IJ, et al. Incidence of community-acquired infections of lower airways among infants. Rev Paul Pediatr. 2016;34:204-209.

6. Michelow IC, Olsen K, Lozano J, et al. Epidemiology and clinical characteristics of community-acquired pneumonia in hospitalized children. Pediatrics. 2004;113(4):701-707.

7. Zar HJ, Cotton MF. Nosocomial pneumonia in pediatric patients: practical problems and rational solutions. Paediatr Drugs. 2002;4(2):73-83.

8. Ostapchuk M, Roberts DM, Haddy R. Community-acquired pneumonia in infants and children. Am Fam Physician. 2004;70(5):899-908.

9. Htoutou Sedláková M, Pudová V, Kolář M. Bacterial pathogens causing hospital-acquired pneumonia - a multicenter study in the Czech Republic. Klin Mikrobiol Infekc Lek. 2015;21:10-14.

10. Cevey-Macherel M, Galetto-Lacour A, Gervaix A, et al. Etiology of community-acquired pneumonia in hospitalized children based on WHO clinical guidelines. Eur J Pediatr. 2009;168(12):1429-1436.

11. Harris M, Clark J, Coote N, et al. British Thoracic Society guidelines for the management of community acquired pneumonia in children: update 2011. Thorax. 2011;66(suppl 2):ii1-ii23.

12. Scott JA, Brooks WA, Peiris JS, Holtzman D, Mulholland EK. Pneumonia research to reduce childhood mortality in the developing world. J Clin Invest. 2008;118(4):1291-1300.

13. Levine OS, Liu G, Garman RL, Dowell SF, Yu S, Yang YH. Haemophilus influenzae type B and Streptococcus pneumoniae as causes of pneumonia among children in Beijing, China. Emerg Infect Dis. 2000; 6(2):165-170.

14. Lu R, Li X, Guo S, et al. Neonatal mortality in the urban and rural China between 1996 and 2013: a retrospective study. Pediatr Res. 2016;79(5):689-696.

15. Clinical and Laboratory Standards Institute. Abbreviated identification of bacterial and yeast; approved guideline. Document m35-a2. 2nd ed. Wayne, PA: Clinical and Laboratory Standards Institute; 2015.

16. Bergogne-Berezin E, Towner KJ. Acinetobacter spp. As nosocomial pathogens: microbiological, clinical, and epidemiological features. Clin Microbiol Rev. 1996;9:148-165.

17. Ngeow YF, Suwanjutha S, Chantarojanasriri T, et al. An Asian study on the prevalence of atypical respiratory pathogens in community-acquired pneumonia. Int J Infect Dis. 2005;9(3):144-153.
18. von Baum H, Welte T, Marre R, Suttorp N, Lück C, Ewig S. Mycoplasma pneumoniae pneumonia revisited within the German competence network for community-acquired pneumonia (CAPNETZ). BMC Infect Dis. 2009;9:62.

19. Shann F. The management of pneumonia in children in developing countries. Clin Infect Dis. 1995;21(supp1 3):S218-S225.

20. Gendrel D, Raymond J, Moulin F, et al. Etiology and response to antibiotic therapy of community-acquired pneumonia in French children. Eur J Clin Microbiol Infect Dis. 1997;16(5):388-391.

21. Ishiwada N, Kurosaki T, Toba T, et al. Etiology of pediatric inpatients with pneumonia. Kansenshogaku Zasshi. 1993;67:642-647.

22. Numazaki K, Chiba S, Umetsu M, et al. Etiological agents of lower respiratory tract infections in Japanese children. In Vivo. 2004;18:67-71.

23. Wubbel L, Muniz L, Ahmed A, et al. Etiology and treatment of community-acquired pneumonia in ambulatory children. Pediatr Infect Dis J. 1999;18(2):98-104.

24. Johnson W, Abdulkarim A. Childhood pneumonia in developing countries. Afr J Respir Med. 2013;8:4-9.

25. Falade AG, Ayede AI. Epidemiology, aetiology and management of childhood acute community-acquired pneumonia in developing countries - a review. Afr J Med Med Sci. 2011;40(4):293-308.

26. Bradley JS, Byington CL, Shah SS, et al. The management of community-acquired pneumonia in infants and children older than 3 months of age: clinical practice guidelines by the pediatric infectious diseases society and the infectious diseases society of America. Clin Infect Dis. 2011;53(7):e25-e76.

27. Otto M. Staphylococcus epidermidis - the 'accidental' pathogen. Nat Rev Microbiol. 2009;7:555-567.

28. Taussig L, Landau L. Pediatric Respiratory Medicine. 2nd ed. Philadelphia: Elsevier Health Sciences; 2008.

29. Webber S, Wilkinson AR, Lindsell D, et al. Neonatal pneumonia. Arch Dis Child. 1990;65:207-211.

30. Li Z, Xiao Z, Li Z, Zhong Q, Zhang Y, Xu F. 116 cases of neonatal early-onset or late-onset sepsis: a single center retrospective analysis on pathogenic bacteria species distribution and antimicrobial susceptibility. Int J Clin Exp Med. 2013;6(8):693-699.

31. Milea D, Azmi S, Reginald P, Verpillat P, Francois C. A review of accessibility of administrative healthcare databases in the Asia-Pacific region. J Mark Access Health Policy. 2015;3(1):28076.
Therapeutics and Clinical Risk Management

\section{Publish your work in this journal}

Therapeutics and Clinical Risk Management is an international, peerreviewed journal of clinical therapeutics and risk management, focusing on concise rapid reporting of clinical studies in all therapeutic areas, outcomes, safety, and programs for the effective, safe, and sustained use of medicines. This journal is indexed on PubMed Central, CAS,
Dovepress

EMBase, Scopus and the Elsevier Bibliographic databases. The manuscript management system is completely online and includes a very quick and fair peer-review system, which is all easy to use. Visit http://www.dovepress.com/testimonials.php to read real quotes from published authors. 